\title{
MAXI J1957+032: An Accreting Neutron Star Possibly in a Triple System
}

\author{
V. Ravi (iD \\ Cahill Center for Astronomy and Astrophysics, MC 249-17, California Institute of Technology, Pasadena, CA 91125, USA; vikram@caltech.edu \\ Received 2017 May 8; revised 2017 October 2; accepted 2017 October 3; published 2017 December 18
}

\begin{abstract}
I present an optical characterization of the Galactic X-ray transient source MAXI J1957+032. This system flares by a factor of $\gtrsim 10^{4}$ every few hundred days, with each flare lasting $\sim 5$ days. I identify its quiescent counterpart to be a late-K/early-M dwarf star at a distance of $5 \pm 2 \mathrm{kpc}$. This implies that the peak $0.5-10 \mathrm{keV}$ luminosity of the system is $10^{36.4 \pm 0.4} \mathrm{erg} \mathrm{s}^{-1}$. As found by Mata Sanchez et al. the outburst properties of MAXI J1957+032 are most consistent with the sample of accreting millisecond pulsars. However, the low inferred accretion rate, and the lack of evidence for a hydrogen-rich accretion flow, are difficult to reconcile with the late-K/early-M dwarf counterpart being the mass donor. Instead, the observations are best described by a low-mass hydrogen- and possibly heliumpoor mass donor, such as a carbon-oxygen white dwarf, forming a tight interacting binary with a neutron star. The observed main-sequence counterpart would then likely be in a wide orbit around the inner binary.
\end{abstract}

Key words: accretion, accretion disks - binaries: general - stars: neutron - X-rays: binaries - X-rays: individual (MAXI J1957+032)

\section{Introduction}

The Monitor of the All-sky X-ray Image (MAXI) experiment on board the International Space Station scans $95 \%$ of the sky at energies between 0.4 and $30 \mathrm{keV}$ during each 92-minute orbit (Matsuoka et al. 2009). The Gas-Slit Camera (GSC), operating between $2-30 \mathrm{keV}$, is the more sensitive of the two MAXI instruments, capable of detecting a $\gtrsim 10$ mCrab source and localizing it to $3 \mathrm{deg}^{2}$ in a single day of observations. One of the primary science goals of the MAXI/GSC instrument is realized through its nova alert system (Negoro et al. 2010), which automatically identifies outbursts in the day-averaged data and provides alerts to the interested community within tens of seconds.

Transient X-ray emission was first identified from a new source, MAXIJ1957+032 (hereafter J1957), by the MAXI/ GSC nova alert system on 2015 May 11 (ATel 7504; Negoro et al. 2015), with a peak flux in the $2-10 \mathrm{keV}$ energy band of $10 \mathrm{mCrab}$. This flare was simultaneously detected by the IBIS/ ISGRI telescope on board the INTEGRAL mission (ATel 7506; Cherepashchuk et al. 2015), with a flux of 4.1(7) mCrab in the 20-60 keV band. ${ }^{1}$ The MAXI flare lasted for two days. A re-brightening of the source was detected by MAXI/GSC on 2015 October 6 (ATel 8143; Sugimoto et al. 2015), and a peak flux of 16(5) mCrab was reached on 2015 October 7 in the $2-20 \mathrm{keV}$ band. Again, the flare lasted for two days in the MAXI energy band. Two further flares were detected by MAXI on 2016 January 7 (ATel 8529; Tanaka et al. 2016) and 2016 September 29 (ATel 9565; Negoro et al. 2016), with comparable fluxes and durations.

J1957 has so far eluded unambiguous characterization. Triggered observations with the Swift X-Ray Telescope (XRT; Burrows et al. 2005) on 2015 May 13, following the first reported MAXI flare, detected a single source within the MAXI/ GSC error circle that faded by a factor of $\sim 100$ over the following week (ATels 7506, 7520; Cherepashchuk et al. 2015; Molkov et al. 2015). A blue optical counterpart coincident with

\footnotetext{
1 Throughout this paper, $1 \sigma$ uncertainties in the last significant figures are provided in parentheses.
}

the Swift/XRT 3" localization was detected by the GROND camera at the MPI/ESO $2.2 \mathrm{~m}$ telescope on 2015 May 15, with a magnitude $r^{\prime}=20.0(1)$, and fading by 1.2 magnitudes by the following night (ATel 7524; Rau et al. 2015). The quiescent optical counterpart was recently identified, with a magnitude $R=21.4(2)$, by the SALTICAM imager at the South African Large Telescope (SALT) (ATel 9649; Buckley et al. 2016). A SALT spectrum of J1957 was obtained following the 2016 September 29 outburst, although only a featureless blue continuum was detected (Buckley et al. 2016; Mata Sánchez et al. 2017). Mata Sánchez et al. (2017) interpreted the spectral properties of J1957 during outburst as corresponding to an X-ray irradiated disk, which, when considered together with the $\mathrm{X}$-ray outburst light curves, were thought to be suggestive of an accreting millisecond X-ray pulsar (AMXP).

I entered the fray following the second reported outburst of J1957 in October 2015, by collating what was known about this source, and attempting to identify the quiescent optical counterpart using deep observations at the W. M. Keck Observatory. On 2016 September 30, I was also able to obtain a spectrum of the optical counterpart to the fourth reported MAXI outburst. A summary and re-analysis of the X-ray observations is presented in Section 2, and the optical observations are presented in Section 3.

The properties of J1957, including the outburst and quiescence timescales, brightness, and detection of optical flares, are most characteristic of a Galactic X-ray binary system. That is, the outbursts are most likely driven by accretion onto a compact object from a companion star. This paper therefore attempts to answer the following questions, in pedagogical order:

1. What is the nature of the quiescent optical counterpart?

2. Is the compact accretor a white dwarf (WD), neutron star (NS), or black hole $(\mathrm{BH})$ ?

3 . How does the accretion occur?

4. What is the evolutionary state of the system?

I present the interpretation in Section 4, and conclude in Section 5. 
Table 1

Summary of MAXI/GSC Detections of Outbursts from MAXI J1957+032

\begin{tabular}{lcc}
\hline \hline Epoch $($ MJD) & $\tau_{f}$ (days) & References \\
\hline 57153 & 2 & $(1),(2),(3),(4)$ \\
$57302(149)^{\mathrm{a}}$ & 2 & $(5),(6),(7),(8)$ \\
$57394(93)^{\mathrm{a}}$ & 3 & $(9)$ \\
$57660(266)^{\mathrm{a}}$ & 2 & $(10)$ \\
\hline
\end{tabular}

Note. (1) ATel 7504, (2) ATel 7506, (3) ATel 7520, (4) ATel 7524, (5) ATel 8143, (6) ATel 8146, (7) ATel 8149, (8) ATel 8197, (9) ATel 8529, (10) ATel 9565.

${ }^{\mathrm{a}}$ The quantities in parentheses are the times since the previous flare.

\section{MAXI J1957+032: X-Ray Observations}

The MAXI/GSC nova alert system has triggered on four outbursts from J1957 to date, as summarized in Table 1. I also list the outburst timescales and relevant references for observations of each outburst. I note that the publicly available MAXI/GSC light curves for the position of J1957 include data since MJD 55058. I encourage the MAXI team to analyze their data on J1957 prior to the first reported flare on MJD 57153, to identify any other outbursts that may not have triggered the nova alert system.

The Swift satellite conducted 28 observations of the position of J1957, in response to the MJD 57153, MJD 57302, and MJD 57660 outbursts. These observations were analyzed by Mata Sánchez et al. (2017). Following each outburst, rapidly fading X-ray emission was detected with the XRT, and rapidly fading UV/optical emission was detected with the UV/Optical Telescope (UVOT; Roming et al. 2005). The maximum detected flux, observed following the MJD 57660 outburst, was 9.1(1) $\times 10^{-10} \mathrm{erg} \mathrm{cm}^{-2} \mathrm{~s}^{-1}$. Mata Sánchez et al. (2017) fitted an absorbed power-law model to the XRT data, finding a largely consistent photon index of $\Gamma \approx 2$, and an absorbing column density of $n_{\mathrm{H}}=1.7 \times 10^{21} \mathrm{~cm}^{-2}$ (see also ATel 9591; Chakrabarty et al. 2016). No periodicities were detected in the X-ray light curves by Mata Sánchez et al. (2017). By stacking the XRT non-detections, Mata Sánchez et al. (2017) placed an upper limit on the quiescent X-ray flux of J1957 of $1-3 \times 10^{-13} \mathrm{erg} \mathrm{cm}^{-2} \mathrm{~s}^{-1}$ (95\% confidence).

I re-analyzed the Swift/XRT data, and confirmed most of the results of Mata Sánchez et al. (2017). The one exception is that I detect J1957 with a flux of $\sim 3 \times 10^{-13} \mathrm{erg} \mathrm{cm}^{-2} \mathrm{~s}^{-1}$ up to 40 days following the MJD 57153 outburst. In Table 2, I present my re-analysis of the XRT observations targeted at J1957 and obtained in the photon-counting mode. Using the HEASOFT distribution v6.21, each observation was reduced using the xrtpipeline task with standard inputs. Images in the $0.5-10 \mathrm{keV}$ band (XRT channels 50-1000) were then extracted using the xselect task. I then used the sosta command of the ximage task to estimate the count rate at the position of J1957. The resulting source count rates were extracted and background-corrected using box sizes that maximized the signal-to-noise ratio, and were additionally corrected for vignetting, exposure, and the point-spread function (PSF) weighted by the xrtpipelinegenerated exposure maps. For observations where no significant emission was detected at the position of J1957, I report $3 \sigma$ upper limits. Finally, all count rates were converted to unabsorbed $0.5-10 \mathrm{keV}$ fluxes assuming an absorbing column of $N_{\mathrm{H}}=1.7 \times 10^{21} \mathrm{~cm}^{-2}$, and a photon index of $\Gamma=1.92$ determined by the weighted average of the measurements of
Table 2

Swift/XRT Photon-counter Observations of MAXI J1957+032

\begin{tabular}{lcccr}
\hline \hline $\begin{array}{l}\text { Epoch } \\
(\mathrm{MJD})\end{array}$ & ObsID & $\begin{array}{c}\text { XRT } \\
\text { Exposure }\end{array}$ & $\begin{array}{c}0.5-10 \mathrm{keV} \\
\text { Count Rate } \\
\left(\mathrm{s}^{-1}\right)\end{array}$ & $\begin{array}{c}0.5-10 \mathrm{keV} \\
\text { unabs Flux } \\
\left(\mathrm{erg} \mathrm{cm}^{-2} \mathrm{~s}^{-1}\right)\end{array}$ \\
\hline 57156.05 & 00033770001 & 2982 & $0.50(1)$ & $1.65(3) \times 10^{-11}$ \\
57157.68 & 00033770002 & 1990 & $0.013(3)$ & $4.2(9) \times 10^{-13}$ \\
57162.07 & 00033770005 & 2783 & $0.006(2)$ & $2.1(6) \times 10^{-13}$ \\
57164.78 & 00033770006 & 867 & $0.008(3)$ & $3(1) \times 10^{-13}$ \\
57165.80 & 00033770007 & 2164 & $0.021(3)$ & $7(1) \times 10^{-13}$ \\
57193.04 & 00033770008 & 4982 & $0.006(1)$ & $2.0(5) \times 10^{-13}$ \\
57304.69 & 00033770009 & 994 & $0.56(3)$ & $1.85(9) \times 10^{-11}$ \\
57305.63 & 00033770010 & 995 & $0.055(9)$ & $1.8(3) \times 10^{-12}$ \\
57306.69 & 00033770011 & 996 & $<0.0385$ & $<1.28 \times 10^{-12}$ \\
57307.72 & 00033770012 & 995 & $<0.0127$ & $<4.22 \times 10^{-13}$ \\
57308.65 & 00033770013 & 1019 & $<0.0384$ & $<1.28 \times 10^{-12}$ \\
57309.28 & 00033770014 & 1043 & $0.004(2)$ & $1.2(7) \times 10^{-13}$ \\
57310.55 & 00033770015 & 754 & $<0.0354$ & $<1.18 \times 10^{-12}$ \\
57311.45 & 00033770016 & 1023 & $<0.0372$ & $<1.24 \times 10^{-12}$ \\
57662.65 & 00033770019 & 392 & $2.42(9)$ & $8.0(3) \times 10^{-11}$ \\
57663.37 & 00033770020 & 1773 & $0.84(3)$ & $2.81(8) \times 10^{-11}$ \\
57664.20 & 00033770021 & 1739 & $0.103(8)$ & $3.4(3) \times 10^{-12}$ \\
57665.62 & 00033770022 & 1402 & $<0.0274$ & $<9.10 \times 10^{-13}$ \\
57667.54 & 00033770023 & 639 & $<0.0175$ & $<5.81 \times 10^{-13}$ \\
57668.11 & 00033770024 & 2040 & $<0.0073$ & $<2.42 \times 10^{-13}$ \\
57669.79 & 00033770025 & 1941 & $<0.0201$ & $<6.68 \times 10^{-13}$ \\
57731.27 & 00033770026 & 8185 & $<0.0023$ & $<7.6 \times 10^{-14}$ \\
\hline & & & &
\end{tabular}

Note. The epoch MJDs are the midpoints of the Swift/XRT exposures. The count rates are corrected for the background, vignetting, exposure, and the point-spread function weighted by the exposure maps. The unabsorbed (unabs) fluxes are calculated assuming a photon index of $\Gamma=1.92$, and an absorbing column of $N_{\mathrm{H}}=1.7 \times 10^{21} \mathrm{~cm}^{-2}$. All upper limits are at the $3 \sigma$ confidence level.

Mata Sánchez et al. (2017). I illustrate the multi-wavelength light curve of the MJD 57153 flare of J1957 in Figure 1.

The cause of the discrepancy between the present work and the non-detection of J1957 between MJD 57157-57193 by Mata Sánchez et al. (2017) is unclear. I additionally find a tentative detection of J1957 on MJD 57309.28 (sosta false alarm probability assuming Poisson statistics of $2.65 \times 10^{-4}$ ). It is possible that the low signal-to-noise ratios of these detections were below the threshold set by Mata Sánchez et al. (2017). Additionally, by stacking the weak detections with non-detections, Mata Sánchez et al. (2017) may have actually reduced the signal-to-noise ratio of the faint emission from J1957 detected on some epochs.

\section{The Optical Counterpart}

I now present observations of the optical counterpart of J1957 at the W. M. Keck Observatory; the observations are detailed in Table 3. I obtained two epochs of imaging of the quiescent source with the Low-Resolution Imaging Spectrometer (LRIS; Oke et al. 1995) on the Keck 1 telescope, and four epochs of spectroscopy with LRIS and the DEep Imaging Multi-Object Spectrograph (DEIMOS; Faber et al. 2003), three of the quiescent counterpart, and one during its active state. The counterpart was detected on all epochs; here I focus on data from Epochs 3-6 from Table 3, because they represent the most sensitive observations. 


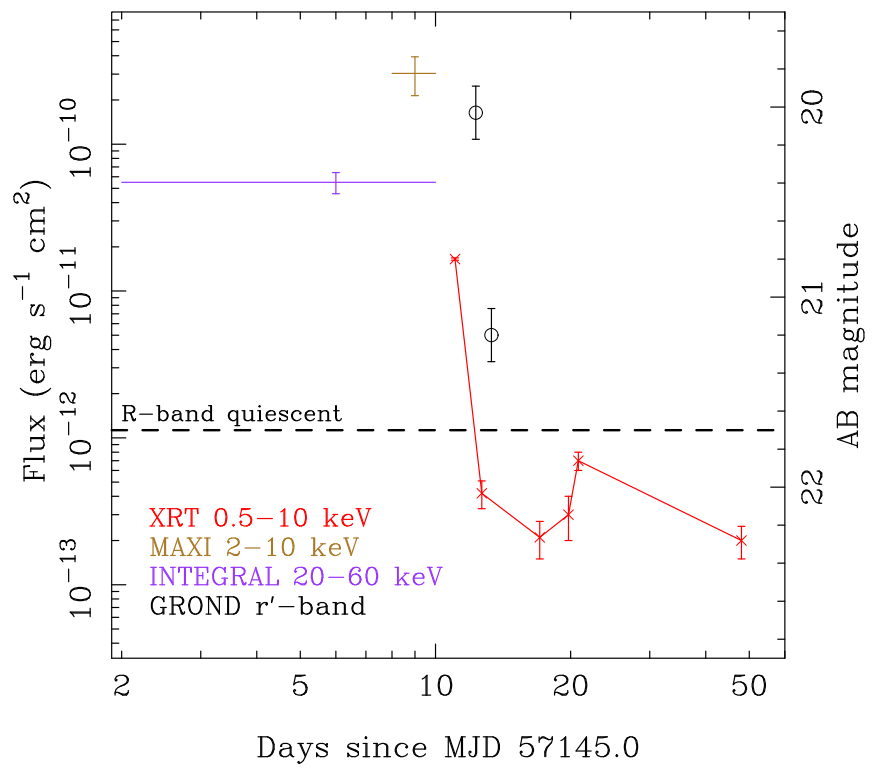

Figure 1. X-ray and optical data on the MJD 57153 flare of MAXI J1957 +032 , reported by $M A X I / G S C$ on 2015 May 11 . The red crosses joined by a curve show the $0.5-10 \mathrm{keV}$ Swift/XRT detections from Table 2 pertinent to this flare. The purple and gold points indicate the times and fluxes of the INTEGRAL 20-60 keV (ATel 7506; Cherepashchuk et al. 2015) and MAXI 2-10 keV (ATel 7504; Negoro et al. 2015) detections respectively. All X-ray fluxes are corrected for absorption. The black open circles show the GROND $r^{\prime}$-band measurements (ATel 7524; Rau et al. 2015), and the dashed line corresponds to the quiescent R-band magnitude measured in the Epoch 5 Keck observations discussed below (Table 3). The X-ray flux scale is on the left ordinate, and the optical flux scale is on the right ordinate.

\subsection{The Active Counterpart}

I obtained a $900 \mathrm{~s}$ spectrum of the optical counterpart with LRIS one day after the MJD 57660 outburst reported by MAXI/GSC. Details on the instrument configuration are in Table 3 (Epoch 6). The slit position angle (PA) was chosen to avoid contamination from a nearby bright star, particularly, the object that confused the authors of ATels 8149 and 8197. The slit orientation is indicated in Figure 2. The data were processed using standard procedures for this instrument. Bias subtraction using the overscan levels, flat-fielding using domeflat exposures, cosmic ray rejection, optimal sky-line subtraction, and wavelength calibration using internal arc exposures corrected by sky-line positions were performed using D. Perley's lpipe software. ${ }^{2}$ Using the trace of the standard star BD $+28^{\circ} 4211$, I then optimally extracted the spectra, and performed telluric absorption line and extinction corrections, flux calibration, and optimal addition of the spectra from the two arms of the spectrograph using the standard spectrum. The seeing was at 0.19 full-width half-maximum (FWHM). The resulting spectrum is displayed in the top panel of Figure 3.

The spectrum is dominated by a smooth blue continuum between 3000 and $10000 \AA$. This is consistent with the results reported by Buckley et al. (2016) and Mata Sánchez et al. (2017) from a SALT spectrum of J1957 obtained approximately one day after these observations. Using the LRIS $R$-band filter profile, I obtain an AB magnitude of 19.47(2), consistent with previous measurements of the optical source in outburst (Rau et al. 2015). In addition to the Na I D doublet in absorption at $\sim 5892 \AA$, a selection of blue metallic emission

\footnotetext{
2 http://www.astro.caltech.edu/ dperley/programs/lpipe.html
}

lines is tentatively detected. These include He II at $3203 \AA$ and $4686 \AA$, S II at $4072 \AA$, and Si II at $5041 \AA$, $6347 \AA$, and $6371 \AA$. These lines were not detected in the SALT observations.

\subsection{The Quiescent Counterpart}

The deepest imaging observations of the quiescent optical counterpart of J1957 were conducted on MJD 57546 (Epoch 5, Table 3). The observations were performed in the LRIS $g, R$, and $I$ bands. The images were bias-subtracted using full-frame bias images, flat-fielded using dome-flat exposures, cleaned of cosmic ray hits, registered astrometrically against USNO B1.0 stars (Monet et al. 2003), and co-added using D. Perley's lpipe software. The seeing FWHM was 0".6. The photometric zeropoint in each filter was derived using same-night observations of SDSS photometric standard fields. I performed manual PSF photometry on the J1957 counterpart. I measured the following AB magnitudes: $g=22.9(1), R=21.7(1), I=21.6(1)$. The I-band image of $\mathrm{J} 1957$ is shown in Figure 2. These measurements are consistent, to within errors, with those reported by Buckley et al. (2016) and Mata Sánchez et al. (2017) of the quiescent counterpart. During the Epoch 2 imaging (Table 3), processing the data using identical techniques, I measured $g=23.5(2)$ and $R=22.3(2)$. Note, however, that although the Epoch 2 data had comparable exposure times, observing conditions were considerably worse and likely not photometric (seeing FWHM of $1^{\prime \prime}$, thin high cirrus cloud).

The best spectroscopic observations of the quiescent counterpart were obtained with LRIS on MJD 57519 (Epoch 3, Table 3). I processed these data exactly as described above for the active counterpart; the standard star was Feige 67, and the seeing FWHM was 0 ". 6 . The resulting spectrum is shown in the bottom panel of Figure 3. Again, using the LRIS $R$-band filter profile, the spectrum is consistent with an $\mathrm{AB}$ magnitude of 22.7(5). However, as with the Epoch 2 imaging data, thin high cirrus cloud present on this night may have made the conditions not suitable for accurate photometry. A red continuum was detected, along with a few absorption features ( $\mathrm{Na}$ I D doublet at $\sim 5892 \AA$, potentially Ca I at $4227 \AA$, and the Ca II doublet at $\sim 7310 \AA$ ). A consistent but lower signal-to-noise ratio spectrum was measured with DEIMOS on MJD 57543; the data were processed using the DEEP2 pipeline. ${ }^{3}$

The broad absorption features at $\sim 5000 \AA$, and between 6000 and $7500 \AA$, are consistent with $\mathrm{MgH}$ and $\mathrm{TiO}$ absorption bands in the atmosphere of a cool star, wherein the $\mathrm{Ca}$ I and $\mathrm{Na}$ I D features are also expected. ${ }^{4}$ I performed a quantitative comparison between the observed spectrum and the Pickles (1998) library of stellar spectra by dividing the measured and template spectra by their continua (modeled as fourth-order polynomials), and measuring a $\chi^{2}$ statistic as a goodness of fit. The best-fitting spectrum was that of a K7V dwarf, with an M0V dwarf also providing an equally good match (factor of 1.01 worse $\chi^{2}$ ). The template continua were somewhat redder than the observed continuum, which may either be intrinsic to the source, or a result of inaccurate spectrophotometric calibration on the night. I show the scaled spectrum of the

\footnotetext{
3 http://deep.ps.uci.edu/spec2d/

4 Although the 5000-5100 $\AA$ band is in fact a blend of $\mathrm{MgH}$ and $\mathrm{TiO}$ absorption features, the extra absorption at the red end of the feature suggests that it is dominated by $\mathrm{MgH}$ absorption (Morgan et al. 1943).
} 
Table 3

Log of Optical Observations

\begin{tabular}{|c|c|c|c|c|c|c|c|c|c|}
\hline Epoch & State $^{\mathrm{a}}$ & Date (MJD) & Instrument & Filter $^{\mathrm{b}}$ & Exposures $^{\mathrm{c}}$ (s) & Slitmask & Slit PA (deg) & Grating/Grism & Magnitude \\
\hline 1 & $\mathrm{Q}$ & 57308.265 & Keck1/LRIS & D560 & $600 ; 600$ & $1^{\prime \prime} \times 175^{\prime \prime}$ & $-45.0^{\mathrm{d}}$ & $400 / 8500$ and $400 / 3400$ & - \\
\hline 2 & $\mathrm{Q}$ & 57362.217 & Keck1/LRIS & $g$ & 120,160 & $\ldots$ & $\ldots$ & $\ldots$ & $23.5(2)^{\mathrm{e}}$ \\
\hline 2 & $\mathrm{Q}$ & 57362.217 & Keck1/LRIS & $R$ & 120,120 & $\ldots$ & $\ldots$ & $\ldots$ & $22.3(2)^{\mathrm{e}}$ \\
\hline 4 & $\mathrm{Q}$ & 57543.512 & $\begin{array}{l}\text { Keck2/ } \\
\text { DEIMOS }\end{array}$ & GG455 & $4 \times 900$ & $1^{\prime \prime} \times 20^{\prime \prime}$ & $47.0^{\mathrm{d}}$ & 600ZD & $\ldots$ \\
\hline 5 & Q & 57546.532 & Keck1/LRIS & $g$ & 30,300 & $\ldots$ & $\ldots$ & $\ldots$ & $22.9(1)$ \\
\hline 6 & A & 57661.265 & Keck1/LRIS & D560 & $900 ; 900$ & $1^{\prime \prime} \times 175^{\prime \prime}$ & 124.5 & $400 / 8500$ and $400 / 3400$ & $R=19.47(2)$ \\
\hline
\end{tabular}

Notes.

a This refers to the optical activity state of the source $(\mathrm{Q}=$ quiescent, $\mathrm{A}=$ active).

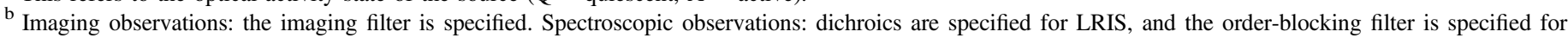
DEIMOS.

${ }^{c}$ Spectroscopic observations: exposure sequences for the LRIS red and blue arms are separated by semi-colons.

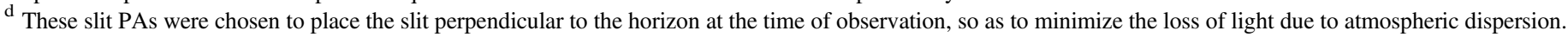

${ }^{\mathrm{e}}$ Obtained under sub-photometric observing conditions, and not inclusive of systematic error of $\sim 0.3$ magnitudes.

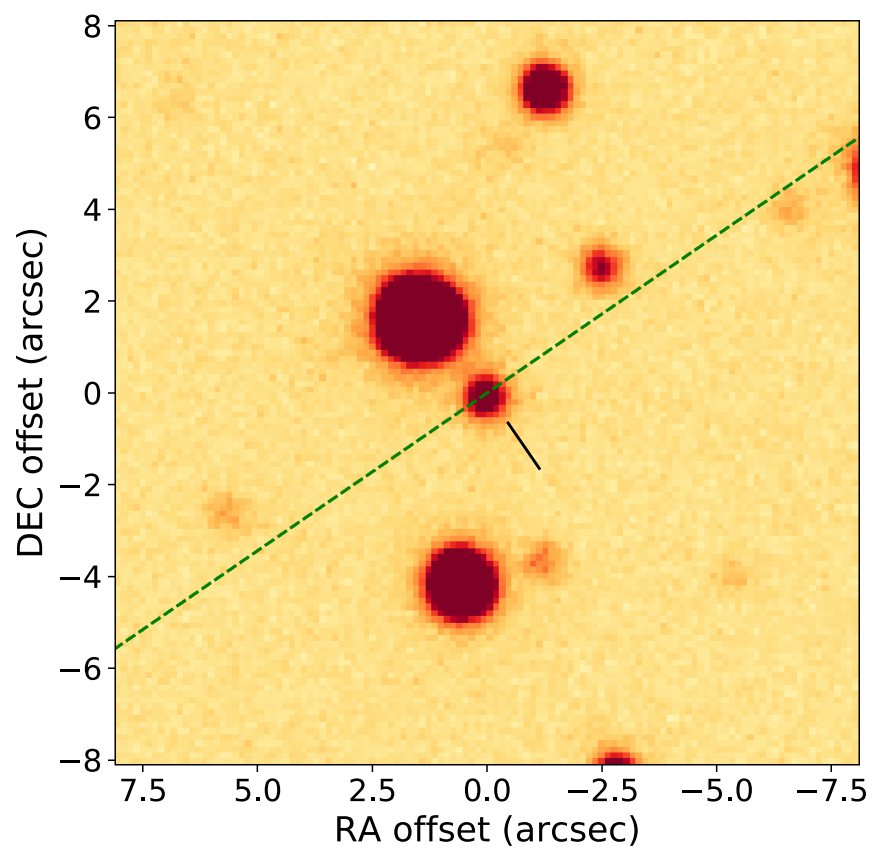

Figure 2. I-band image of the quiescent optical counterpart to J1957 from the Epoch 5 observations (Table 3 ). The counterpart is indicated by a black solid line. The image is centered on the position of J1957 (J2000): $19^{\mathrm{h}} 56^{\mathrm{m}} 39^{\mathrm{s}} .11,03^{\circ} 26^{\prime} 43^{\prime \prime}$. $^{\circ}$. The green dashed line indicates the slit PA (124.5) during the spectroscopic observations of Epochs 3 and 6.

Pickles (1998) K7V dwarf in Figure 3. Giants of a similar spectral type (i.e., K7III, M0III) also provided a good fit (factor of 1.05 worse $\chi^{2}$ ), and it is thus difficult to distinguish between a cool dwarf and giant object spectroscopically.

However, it is substantially more likely that the counterpart is a dwarf object than a giant. Late- $\mathrm{K}$ to early-M giants have absolute magnitudes brighter than $R=1$ (Cox 2000). J1957 is at Galactic coordinates $l=43^{\circ} .65, b=-12.83$, and I thus adopt a maximum distance of $20 \mathrm{kpc}$ (assuming that it lies within the Milky Way disk), implying a distance modulus of $<16.5$ magnitudes. If the counterpart were a giant, the $R$-band extinction along its sightline must be $A_{R}>4$ magnitudes for it to be consistent with our brightest apparent magnitude measurement. Let us now make the assumption that $A_{V} \approx A_{R}$ to estimate a lower limit on $E(B-V)$; this is conservative, as typically $A_{V} \gtrsim A_{R}$. Then, the $A_{R}>4$ constraint implies $E(B-V)>1.3$ under typical Milky Way conditions. The expected Milky Way extinction along this sightline is characterized by $A_{R}=0.27, A_{V}=0.34$, and $E(B-V)=$ 0.11 (Schlafly \& Finkbeiner 2011).

Using the relation between the equivalent width of the $\mathrm{Na}$ I D doublet $\left(\mathrm{EW}_{\mathrm{Na} \text { I }}\right)$ and extinction identified by Poznanski et al. (2012) for the Milky Way, I find $\mathrm{EW}_{\mathrm{Na} \text { I D }}>1.7 \AA$. Even if the conservative assumption were made that the blue continuum of the active counterpart to J1957 has no intrinsic Na I absorption, the measured equivalent width of the absorption feature is only 0.5 (2) $\AA$. This measurement is in fact consistent with the Schlafly \& Finkbeiner (2011) expectation for this sightline through the Milky Way. Furthermore, using the relation between $A_{V}$ and $n_{\mathrm{H}}$ identified by Güver \& Özel (2009), an H I column of $n_{\mathrm{H}}>8.8 \times 10^{21} \mathrm{~cm}^{-2}$ would be expected. Such a large column is not evident in the X-ray spectrum of J1957 (Chakrabarty et al. 2016; Mata Sánchez et al. 2017).

I therefore classify the quiescent counterpart to J1957 as a dwarf star of late-K or early-M spectral type. Note that these arguments are based on the assumption that the extinction does not significantly vary between active and quiescent states. Additionally, it is unlikely that the dwarf star is present at the position of J1957 by chance. The only optical image of J1957 in its active state was obtained by Rau et al. (2015), and their position is consistent with the centroid of the quiescent counterpart to within 0.13 , which is within the combined astrometric error of the Rau et al. (2015) measurements and our astrometric solution (both are individually correct to 0 !"2). The mean surface density of all detected objects in the Epoch 5 $R$-band image is $\approx 0.03 \operatorname{arcsec}^{-2}$, making a chance coincidence very unlikely $(\approx 1 / 300$ for any object in our image). I hence proceed under the assumption that the late-K/early-M dwarf is physically associated with the J1957 system. 


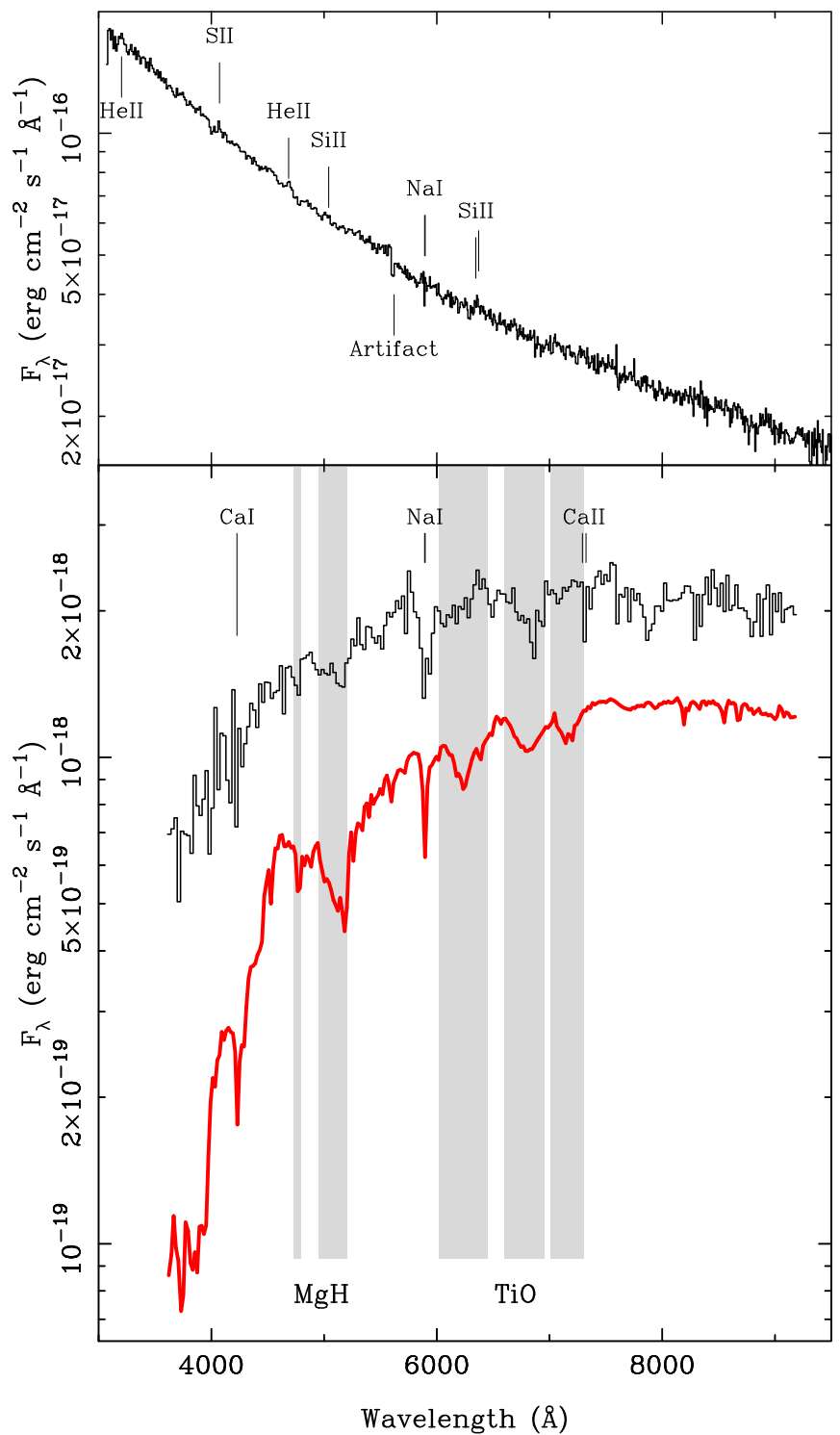

Figure 3. Spectra of the optical counterpart to MAXI J1957+032 during an outburst (top-Epoch 6) and quiescence (bottom-Epoch 3). Relevant spectral lines and molecular absorption bands are indicated. The measured spectra are shown as binned data, and a template spectrum of a K7V dwarf from the Pickles (1998) spectral atlas is shown as a thick red trace in the bottom panel. The template is arbitrarily scaled in flux density for convenience of display. The quiescent spectrum shares numerous features with the K7V template, including the $\mathrm{Na}$ I and $\mathrm{Ca}$ I narrow absorption features, as well as the broad $\mathrm{MgH}$ and $\mathrm{TiO}$ absorption bands. However, although this template is the best fit to the data from the Pickles atlas, it is apparent that the quiescent spectrum is bluer than the template. No interstellar extinction correction was applied to the data; the total Milky Way extinction along the J1957 sightline is expected to be $E(B-V)=0.11$

\section{Interpretation}

\subsection{Empirical Results}

Identification of the counterpart to J1957 permits an estimate of the distance to the system. The fourth edition of Allen's Astrophysical Quantities (Cox 2000) lists an absolute $V$-band magnitude of 8.8 for M0V stars, ${ }^{5}$ and a color of $V-R=1.28$. I estimated the uncertainty in the absolute magnitude by inspecting

\footnotetext{
5 No listing is present for K7V stars. I therefore assume an M0V spectral type for the absolute magnitude estimate.
}

Table 4

Properties of J1957

\begin{tabular}{lc}
\hline \hline Quantity & Value \\
\hline$L_{\mathrm{XP}}$ & $10^{36.4(4)} \mathrm{erg} \mathrm{s}^{-1}$ \\
$L_{\mathrm{XQ}}$ & $10^{32.7(5)} \mathrm{erg} \mathrm{s}^{-1}$ \\
$L_{R}$ & $10^{32.1(4)} \mathrm{erg} \mathrm{s}^{-1}$ \\
$D C$ & 0.03 \\
\hline
\end{tabular}

the publicly available HIPPARCOS catalog (Perryman et al. 1997). Following Figure 3 of Perryman et al. (1997), I extracted the $V$-band magnitude and parallax measurements for all M0V stars with fractional errors in the parallax of $<10 \%$, calculated absolute magnitudes, and found a $1 \sigma$ uncertainty of 0.8 magnitudes. An exact estimate of the absolute magnitudes of M0V stars from the parallax data is beyond the scope of this work, as such estimates are subject to significant biases (e.g., Oudmaijer et al. 1999). I therefore adopt an absolute (color-corrected) $R$-band magnitude for the J1957 counterpart of 7.5(8).

I use the Epoch 5 R-band magnitude of 21.7(1) for the J1957 counterpart to estimate its distance, as this epoch provided the most accurate photometric measurements. The H I column density of $n_{\mathrm{H}}=1.7 \times 10^{21} \mathrm{~cm}^{-2}$ (Mata Sánchez et al. 2017) implies a $V$-band extinction of $A_{V}=0.77$ (3) (Güver \& Özel 2009), and thus $A_{R}=0.61(2)$ (Schlafly \& Finkbeiner 2011). This is somewhat larger than expected along this Milky Way sightline (Schlafly \& Finkbeiner 2011). The final distance estimate for the J1957 system is 5(2) kpc. To illustrate the sensitivity of this estimate to the assumed extinction, disregarding extinction would result in a distance of $6.9 \mathrm{kpc}$, and an H I column of $n_{\mathrm{H}}=4 \times 10^{21} \mathrm{~cm}^{-2}$ would imply a distance of $3.6 \mathrm{kpc}$.

Having established a distance to J1957, the rest-frame properties of the source can be derived. These include the peak measured $0.5-10 \mathrm{keV}$ X-ray luminosity, $L_{\mathrm{XP}}$, in the Swift/XRT windowed-timing data on MJD57660.69 (Mata Sánchez et al. 2017), the lowest from 0.5 to $10 \mathrm{keV}$ X-ray luminosity measurement, $L_{\mathrm{XQ}}$, from the Swift/XRT detection on MJD 57193 (Table 2), and the peak measured $R$-band luminosity, $L_{R}$. I list these properties in Table 4 . I also include an estimate of the activity duty cycle, $D C$, assuming (based on the MJD 57153 flare) five days of activity per outburst.

Despite large uncertainties in the parameter estimates, we now have a basis for comparing J1957 with known classes of Galactic X-ray transients. Although an exotic extragalactic origin for J1957 may be possible, if, for example, a background galaxy were confused with the quiescent stellar counterpart, I see no reason to consider such an unusual interpretation. Furthermore, the tentatively detected optical emission lines during the most recent $\mathrm{J} 1957$ outburst are not redshifted.

J1957 shares many properties of low-mass X-ray binaries (LMXBs), with transient episodes of high accretion rates from a Roche lobe overflowing star onto a compact object (NS or stellar-mass $\mathrm{BH}$ ), known widely as X-ray novae (for a review, see Tanaka \& Shibazaki 1996). These systems exhibit outbursts of a few weeks to a few months, with duty cycles of $\sim 10 \%$, and with X-ray to optical flux ratios of $\sim 10^{2}-10^{3}$. Although the nondetection of strong emission lines (in particular the Balmer series of hydrogen) during outburst is unusual (van Paradijs \& McClintock 1995), it is not exceedingly rare for a single-epoch observation (Degenaar et al. 2016). The peak X-ray luminosity of J1957 is low with respect to the X-ray nova population; X-ray 
novae typically peak at $>10^{37} \mathrm{erg} \mathrm{s}^{-1}$, a factor of $\sim 100$ greater than is observed for J1957. The outburst timescales, times between outbursts, and duty cycle of J1957 are also small with respect to the X-ray nova population. The faint-state X-ray luminosity, $L_{\mathrm{XQ}}$, is larger by a factor of a few hundred than has been observed for a BH LMXB (e.g., Bildsten \& Rutledge 2000).

As stated by Mata Sánchez et al. (2017), the characteristic outburst duration, light-curve shape, featureless optical spectrum, and duty cycle of J1957 are consistent with the sample of known AMXPs. The AMXP population (Patruno \& Watts 2012) includes objects such as NGC 6440 X-2 (Altamirano et al. 2010), with short outburst timescales of 3-5 days and recurrence times as short as 30 days, and a maximum $2-10 \mathrm{keV}$ luminosity of $1.7 \times 10^{36} \mathrm{erg} \mathrm{s}^{-1}$. In general, ultracompact AMXPs with orbital periods $P_{\text {orb }}<80$ minutes, and partially or fully degenerate companions, exhibit outbursts as faint, short, and often as J1957. Although Mata Sánchez et al. (2017) did not detect pulsations at frequencies in the range $0.1-281 \mathrm{~Hz}$ from $\mathrm{J} 1957$ during its outbursts, several AMXPs spin more rapidly and do not always exhibit pulsations (Patruno \& Watts 2012).

However, although the luminosity I derive for J1957 based on the counterpart identification is consistent with AMXP outbursts, the main-sequence nature of the counterpart raises some questions. All AMXPs with main-sequence mass donors have exhibited thermonuclear Type-I X-ray bursts (Patruno \& Watts 2012), while none have been observed in J1957. For a late-K/early-M dwarf companion, a lower main-sequence equation of state can be assumed, where the counterpart mass is given by $M_{c} / M_{\odot} \approx 0.6$. This would be among the largest companion masses in AMXPs, comparable with Swift J1749.4 -2807 (Altamirano et al. 2011). The variability I detect in the $\mathrm{X}$-ray flux during the times between outbursts also requires an explanation. In particular, the source does not appear to fully fade after the MJD 57153 outburst, but fades to a much greater extent following the MJD57302 and MJD 57660 outbursts. Such variability in the putative quiescent state has only been observed in the AMXP IGR J18245-2452 (Linares et al. 2014), a system that transitions between a radio-pulsar state and AMXP-like outbursts. Below, I address these questions through theoretical considerations.

\subsection{Theoretical Insight}

\subsubsection{The Accretion Flow Model}

I first characterize the ratio, $\Lambda$, between the luminosity of J1957 and the Eddington (1916) luminosity. For ionized hydrogen plasma, this is given by

$$
\Lambda=8 \times 10^{-3} \frac{L_{X}}{10^{36} \operatorname{erg~s}^{-1}}\left(\frac{M}{M_{\odot}}\right)^{-1},
$$

where $M$ is the mass of the compact object. Note that $\Lambda$ decreases for plasmas with significant metal fractions; a pure helium plasma, for example, would correspond to a factor of four decrease in $\Lambda . \Lambda$ also represents the ratio of the mass accretion rate, $\dot{M}$, to the Eddington-limiting mass accretion rate $\left(\dot{M}_{\text {Edd }}\right)$, irrespective of the radiative efficiency of the system. Hence, it appears that at the maximum of the X-ray light curve, J1957 accretes at $\dot{M} \approx 0.014 \dot{M}_{\text {Edd }}$ for a $1.4 M_{\odot}$ NS primary. I note that the correct X-ray luminosity to use in Equation (1) when inferring the instantaneous ratio $\dot{M} / \dot{M}_{\text {Edd }}$ is the instantaneous luminosity, not the time-averaged luminosity that I use below to derive the mean mass-loss rate from the secondary. I assume that the X-ray luminosity is approximately equivalent to the bolometric luminosity.

The low Eddington ratio $\Lambda$ fully excludes a $\mathrm{BH}$ accretor in J1957. At low Eddington ratios, accretion in the standard cool, optically thick accretion disk model (Shakura \& Sunyaev 1973) is less likely to be stable than accretion in a hot, optically thin advection-dominated accretion flow (ADAF; Narayan \& $\mathrm{Yi}$ 1995). If the primary in $\mathrm{J} 1957$ is a $\mathrm{BH}$, likely of mass $M \gtrsim 6 M_{\odot}$ (i.e., as massive as, or more massive than the accretor in GROJ1655-40, which is the smallest mass confirmed for a stellar-mass BH; Corral-Santana et al. 2016), $\Lambda \lesssim 3.3 \times 10^{-3}$. ADAFs are expected for BHs accreting at $\Lambda \lesssim \alpha^{2}$, where $\alpha \approx 0.1$ is the accretion flow viscosity parameter (Narayan \& Yi 1995). However, an ADAF configuration for the accretion implies that a much larger optical luminosity, of the same order of magnitude as the X-ray luminosity, would then be expected. This is not the case in J1957 (see also Mata Sánchez et al. 2017).

On the other hand, ADAFs are only expected for NSs accreting at $\Lambda \lesssim 0.1 \alpha^{2} \approx 10^{-3}$. Therefore, disk accretion is likely for the bright states of J1957. This is not the case for the faint-state detections of J1957, wherein $\Lambda \approx 2.9 \times 10^{-6}$. However, quiescent NS LMXBs can emit X-rays through a variety of mechanisms, including thermal emission from the NS surface, and non-thermal magnetospheric emission (e.g., Campana et al. 2008; Linares et al. 2014). Chromospheric activity in the main-sequence counterpart is unlikely due to the lack of expected optical emission-line features during quiescence (e.g., Ca II H and K, Balmer-series hydrogen).

\subsubsection{The Mass Accretion Rate}

I now consider the implications of the time-averaged mass accretion rate of the $\mathrm{J} 1957$ primary. Assuming a typical outburst bolometric luminosity of $10^{36} \mathrm{erg} \mathrm{s}^{-1}$, a duty cycle of 0.03 , a typical quiescent luminosity of $10^{32} \mathrm{erg} \mathrm{s}^{-1}$, and that the radiation is isotropic, the time-averaged mass accretion rate is

$$
\dot{M} \approx 5 \times 10^{-13}\left(\frac{1}{\eta}\right) M_{\odot} \mathrm{yr}^{-1}
$$

Here, $\eta$ is the time-averaged (and luminosity-weighted) efficiency of the conversion between accreted mass and radiated energy. This, however, is clearly a lower limit. An upper limit under the same assumptions can be derived by assuming that only the gravitational potential energy of the accretion flow once at the compact object surface is converted to radiation. Then, for an NS accretor,

$$
\dot{M} \lesssim 3 \times 10^{-12} \frac{R}{10 \mathrm{~km}}\left(\frac{M}{1.4 M_{\odot}}\right)^{-1} M_{\odot} \mathrm{yr}^{-1},
$$

where $R$ is the NS radius.

I compare these estimates with the expected Roche lobe overflow mass-loss rate for a late-K/early-M dwarf star, following arguments by King (1988). Assuming a lower main-sequence equation of state for the star as before, the stellar radius is given by $R_{c} / R_{\odot}=M_{c} / M_{\odot} \approx 0.6$. Then, equating the stellar radius with the size of its Roche lobe, 
and assuming a circular Keplerian orbit, the orbital period is $P_{\text {orb }} \approx 6 \mathrm{hr}$ (King 1988, Equation (4)). The minimum Roche lobe overflow mass-loss rate can then be estimated by ascribing the shrinking of the Roche lobe to orbital decay driven only by gravitational radiation. From Equation (14) of King (1988), this is given by $\dot{M} \gtrsim 5 \times 10^{-11} M_{\odot} \mathrm{yr}^{-1}$. Therefore, a standard Roche-lobe overflow configuration for J1957 is unlikely for an NS accretor, if the mass donor is the late-K/early-M dwarf star. This result is robust to a factor of 10 uncertainty in the luminosity.

Other mass-loss mechanisms for the late-K/early-M dwarf are also unlikely to feed an NS primary at the inferred accretion rate. Based on analyses of metal-rich WD atmospheres where M dwarf companions are present, Debes (2006) suggested wind mass-loss rates of $10^{-16}-10^{-14} M_{\odot} \mathrm{yr}^{-1}$ for M-dwarfs. Through the analysis of "astrospheric" Ly $\alpha$ absorption features from the collision regions between stellar winds and the interstellar medium, dwarf main-sequence stars are found to have comparable mass-loss rates to the Sun (i.e., $\approx 10^{-14} M_{\odot} \mathrm{yr}^{-1}$; Wood et al. 2005). Somewhat higher mass-loss rates may be expected if X-ray irradiation of the secondary by an NS primary significantly affects the surface temperature. However, assuming a $3 \mathrm{hr}$ orbit for the system, and a quiescent luminosity of J1957 due to thermal emission from the surface of an NS primary, $\approx 10^{31} \mathrm{erg} \mathrm{s}^{-1}$ will be incident on the companion. Thermal equilibrium suggests that this would raise the surface temperature by $\sim 10^{3} \mathrm{~K}$. A detailed model for the effects of X-ray irradiation on companions in LMXBs was presented by Hameury et al. (1986), who showed that the instantaneous accretion rate cannot be expected to be steady due to irradiation effects for binary periods $\lesssim 3 \mathrm{hr}$. However, a time-averaged accretion rate of $>10^{-13} M_{\odot} \mathrm{yr}^{-1}$ is unlikely to be sustained through this mechanism.

\subsection{An Unidentified Mass Donor?}

It is possible that the quiescent counterpart of J1957 is not in fact the star from which the compact object is accreting. This could be the case in two scenarios: (a) if the late-K/early-M dwarf is at the sky-position of J1957 by chance, or (b) if the dwarf is part of the J1957 system, but forms a wide triple with a compact interacting binary. I have already rejected possibility (a), and I therefore adopt possibility (b).

In this scenario, the most compelling candidate for the donor object would be a degenerate star, likely low-mass, in a tight orbit with the primary. Two ways of achieving the required low mass-transfer rates have been suggested for low-luminosity transients like J1957. First, once an LMXB has evolved to an orbital period of $\sim 80$ minutes where the donor reaches a mass of $\lesssim 0.1 M_{\odot}$ and becomes partially degenerate, the mass-transfer rate is slowed to $\lesssim 10^{-11} M_{\odot} \mathrm{yr}^{-1}$ (King 2000). The orbital period increases after this point, as further mass-loss causes the donor to expand. This state is stable for many Gyr, and such systems are expected to be transients owing to the thermalviscous disk instability and the low mass-transfer rates, and dominated by NS accretors because of the predominance of NSs in all LMXBs (transient or persistent). Second, similar behavior is expected for ultracompact LMXBs that are the NS analogs of AM CVn systems (Hameury \& Lasota 2016), with the main distinguishing feature from the first possibility being a somewhat higher-mass degenerate companion, and an orbital period below $\sim 80$ minutes.
Objects in both the configurations mentioned above are expected to be common in the Milky Way, and are the leading candidates to explain X-ray transients with low inferred masstransfer rates where no optical counterparts are identified (Muno et al. 2005). An attractive feature of both models is the expected lack of Balmer-series hydrogen or HeI emission lines in the spectrum of J1957 during outburst. These species are generally observed in LMXB outbursts (e.g., van Paradijs \& McClintock 1995), and may be expected to be present if the donor star in J1957 were a late-K/early-M dwarf as assumed above (although not necessarily for short-period binaries; Degenaar et al. 2016). The outburst characteristics of J1957 are in fact quite similar to those of the transient ultracompact LMXB candidate 1RXS J180408.9-34205 (Baglio et al. 2016; Degenaar et al. 2016). Indeed, the INTEGRAL hard X-ray detection of J1957 during the MJD 57153 outburst that possibly preceded the MAXI detection at softer energies may have represented a hard state of the system that transitioned to a soft state around the epoch of the MAXI observations, in analogy with the outburst of 1RXS J180408.9-34205. Furthermore, the spectrum of 1RXS J180408.9-34205 during outburst is similarly featureless like that of J1957, with the tentative detection of the $4686 \AA \mathrm{He}$ II line in emission.

A second attractive feature of these models is the expectation of no thermonuclear bursts. For a pure helium or carbonoxygen WD donor, no hydrogen is expected to be present in the accreting material. Additionally, the AMXP IGR 00291 +5934 with a brown dwarf companion has also not displayed Type-I bursts, although this is not the case for two other AMXPs with brown dwarf companions (Patruno \& Watts 2012).

A white dwarf or brown dwarf donor in the J1957 system would not be detected in my observations during quiescence. Typical WDs are approximately two to four magnitudes less luminous than the luminosity I assume for the early-K/late-M dwarf star, and brown dwarfs are several magnitudes fainter still.

\subsection{Summary of Interpretation}

I propose that $\mathrm{J} 1957$ consists of an NS in a compact $\left(P_{\text {orb }} \lesssim 2 \mathrm{hr}\right.$ ) mass-transferring binary system, with a hydrogenand possibly helium-poor donor. A carbon-oxygen WD is preferred as the mass donor from a comparison with other accreting NSs. The optical counterpart identified during quiescence is then likely to be in a wide orbit around the binary; a few-hundred-days orbital period is preferred due to stability considerations (Mardling \& Aarseth 2001).

Approximately $20 \%$ of multiple-star systems are found in triples (Eggleton \& Tokovinin 2008), and two NSs in triple systems are known (Garcia et al. 1989; Ransom et al. 2014). One of these systems is the LMXB 4U 2129+47, which consists of an NS accreting from a main-sequence dwarf star in a $4.27 \mathrm{hr}$ orbit, with an additional F7V star in a $>175$ day orbit (Garcia et al. 1989; Bothwell et al. 2008). As the orbital period of the interacting binary is smaller than a day, the binary will likely shrink through the loss of energy and angular momentum to magnetic-dipole and gravitational radiation to form an ultracompact LMXB with a degenerate donor (Pylyser \& Savonije 1988; van Haaften et al. 2013). Such a system, but with a lower-mass tertiary, would be analogous to the scenario that I favor for J1957. 
The formation mechanisms and rates of NS triple systems such as the one proposed for J1957 have been investigated by Freire et al. (2011) and Portegies Zwart et al. (2011). Accreting NSs in hierarchical triples are the leading candidate progenitors for the formation of millisecond pulsars observed in wide binaries with main-sequence companions, including PSR J1903 +0327 (Champion et al. 2008) and PSR J1024-0719 (Bassa et al. 2016; Kaplan et al. 2016). These systems are posited to have begun as hierarchical main-sequence stellar triples, where the most massive component underwent a supernova and formed an NS. The pre-supernova common envelope evolution phase is expected to have shrunk the initial orbits, such that after the supernova the inner main-sequence star can fill and overflow its Roche lobe. If the initial orbit upon mass-transfer is sufficiently large, further evolution of the mass donor will cause the orbit to grow, and dynamical instabilities will eventually lead to its ejection from the system. On the other hand, if the initial orbit were sufficiently small, the interactingbinary orbit could shrink as described above, and the mass donor would evolve off the main sequence, forming a system like J1957. The mass donor may then be ablated by the NS once it has gained sufficient angular momentum to form a millisecond pulsar.

\section{Summary and Future Prospects}

MAXIJ1957+032 (J1957) is an unusual Galactic X-ray transient. It flares by a factor of $\gtrsim 10^{4}$ once every few hundred days, with each flare typically lasting just $\approx 5$ days. I have identified its quiescent optical counterpart to be a late-K/early$\mathrm{M}$ dwarf star, and thus establish the distance to the system to be 5 (2) kpc. An optical spectrum obtained during a flare reveals a blue continuum with weak metallic emission lines.

The distance to J1957 implies a peak $0.5-10 \mathrm{keV}$ luminosity of $10^{36.4(4)} \mathrm{erg} \mathrm{s}^{-1}$. Faint X-ray emission with a luminosity of $10^{32.7(5)} \mathrm{erg} \mathrm{s}^{-1}$ is detected on some epochs between flares, with upper limits that are a few times lower on other epochs. As also found by Mata Sánchez et al. (2017), the outburst behavior of $\mathrm{J} 1957$ is most consistent with the accreting millisecond X-ray pulsar (AMXP) sample. However, the low time-averaged mass accretion rate that I infer of approximately $10^{-12} M_{\odot} \mathrm{yr}^{-1}$ is difficult to reconcile with the Roche-lobe overflow of the late$\mathrm{K} /$ early-M dwarf counterpart, if this counterpart is indeed the mass donor. Further pieces of circumstantial evidence argue against a main-sequence mass donor: no Balmer-series hydrogen or HeI emission lines are present in the optical spectrum of J1957 in its flaring state, and no thermonuclear Type-I X-ray bursts have been detected. Therefore, I hypothesize that the mass donor is not detected in the optical observations I present, but is instead a degenerate hydrogenand possibly helium-poor star in a tight binary system with the NS. A comparison with the AMXP sample (Patruno \& Watts 2012) suggests an ultracompact ( $P_{\text {orb }}<80$ minutes) binary with a carbon-oxygen WD mass donor as the preferred candidate. In this case, J1957 is likely to be a triple system, with the observed main-sequence counterpart in a wide orbit around the mass-transferring binary. Such systems are predicted to exist along the leading formation channel for millisecond pulsars observed in wide binaries with mainsequence companions (Freire et al. 2011; Portegies Zwart et al. 2011).

To make progress toward a better observational understanding of J1957, a measurement of the orbital period of the system, and a direct determination of the nature of the mass donor are crucial. The most valuable observations would be multiple epochs of optical spectroscopy during an outburst. The emission lines that were observed in my Epoch 6 data, if indeed present, are clearly comparable in flux density to the hot thermal continuum. Their equivalent widths would increase if the accreting gas is allowed to cool, which is highly likely to occur as the system returns to quiescence. Thus, late-time optical spectroscopy of an outburst can yield more information on the composition of the accretion flow. The presence of hydrogen is expected in the case of a late-K/early-M dwarf donor. If the gas temperature during my observations was $\gtrsim 2 \times 10^{4} \mathrm{~K}$, under reasonable pressures it is unlikely that Balmer-series hydrogen lines could have been observed. However, these lines would be expected at later times. Moderate-resolution ( 3 A FWHM) time-resolved spectroscopy would also provide sensitivity to any orbital period $\lesssim 10 \mathrm{hr}$ through Doppler shifts of spectral lines. If the late-K/early-M dwarf star is the mass donor, an orbital period of more than a few hours may be expected. A somewhat shorter orbital period would be observed if the system is an ultracompact LMXB in a triple system with the main-sequence star.

I thank S. Kulkarni for introducing me to this object, N. Blagorodnova, Y. Cao, G. Duggan, and R. Lunnan for assistance with the optical observations, and S. Phinney and T. Maccarone for useful discussions. The data presented herein were obtained at the W. M. Keck Observatory, which is operated as a scientific partnership among the California Institute of Technology, the University of California and the National Aeronautics and Space Administration. The Observatory was made possible by the generous financial support of the W. M. Keck Foundation. I recognize and acknowledge the very significant cultural role and reverence that the summit of Maunakea has always had within the indigenous Hawaiian community. I acknowledge the use of public data from the Swift data archive. This research has made use of the VizieR catalog access tool, CDS, Strasbourg, France.

Facilities: Keck:I (LRIS), Keck:II (DEIMOS), MAXI, Swift.

\section{ORCID iDs}

V. Ravi (i) https://orcid.org/0000-0002-7252-5485

\section{References}

Altamirano, D., Cavecchi, Y., Patruno, A., et al. 2011, ApJL, 727, L18 Altamirano, D., Patruno, A., Heinke, C. O., et al. 2010, ApJL, 712, L58 Baglio, M. C., D’Avanzo, P., Campana, S., et al. 2016, A\&A, 587, A102 Bassa, C. G., Janssen, G. H., Stappers, B. W., et al. 2016, MNRAS, 460, 2207 Bildsten, L., \& Rutledge, R. E. 2000, ApJ, 541, 908

Bothwell, M. S., Torres, M. A. P., Garcia, M. R., \& Charles, P. A. 2008, A\&A, 485,773

Buckley, D. A. H., Kotze, M. A., Charles, P. A., et al. 2016, ATel, 9649, 1 Burrows, D. N., Hill, J. E., Nousek, J. A., et al. 2005, SSR, 120, 165 Campana, S., Stella, L., \& Kennea, J. A. 2008, ApJL, 684, L99

Chakrabarty, D., Jonker, P. G., \& Markwardt, C. B. 2016, ATel, 9591, 1 Champion, D. J., Ransom, S. M., Lazarus, P., et al. 2008, Sci, 320, 1309 Cherepashchuk, A. M., Molkov, S. V., Lutovinov, A. A., \& Postnov, K. A. 2015, ATel, 7506, 1

Corral-Santana, J. M., Casares, J., Muñoz-Darias, T., et al. 2016, A\&A, 587, A61

Cox, A. N. 2000, Allen's Astrophysical Quantities (IV ed.; New York: AIP) Debes, J. H. 2006, ApJ, 652, 636

Degenaar, N., Altamirano, D., Parker, M., et al. 2016, MNRAS, 461, 4049 Eddington, A. S. 1916, MNRAS, 77, 16 
Eggleton, P. P., \& Tokovinin, A. A. 2008, MNRAS, 389, 869

Faber, S. M., Phillips, A. C., Kibrick, R. I., et al. 2003, Proc. SPIE, 4841, 1657

Freire, P. C. C., Bassa, C. G., Wex, N., et al. 2011, MNRAS, 412, 2763

Garcia, M. R., Bailyn, C. D., Grindlay, J. E., \& Molnar, L. A. 1989, ApJL, 341, L75

Güver, T., \& Özel, F. 2009, MNRAS, 400, 2050

Hameury, J. M., King, A. R., \& Lasota, J. P. 1986, A\&A, 162, 71

Hameury, J.-M., \& Lasota, J.-P. 2016, A\&A, 594, A87

Kaplan, D. L., Kupfer, T., Nice, D. J., et al. 2016, ApJ, 826, 86

King, A. R. 1988, QJRAS, 29, 1

King, A. R. 2000, MNRAS, 315, L33

Linares, M., Bahramian, A., Heinke, C., et al. 2014, MNRAS, 438, 251

Mardling, R. A., \& Aarseth, S. J. 2001, MNRAS, 321, 398

Mata Sánchez, D., Charles, P. A., Armas Padilla, M., et al. 2017, MNRAS, 468, 564

Matsuoka, M., Kawasaki, K., Ueno, S., et al. 2009, PASJ, 61, 999

Molkov, S. V., Lutovinov, A. A., Postnov, K. A., \& Cherepashchuk, A. M. 2015, ATel, 7520, 1

Monet, D. G., Levine, S. E., Canzian, B., et al. 2003, AJ, 125, 984

Morgan, W. W., Keenan, P. C., \& Kellman, E. 1943, An Atlas of Stellar Spectra, with an Outline of Spectral Classification (Chicago, IL: Univ. Chicago Press)

Muno, M. P., Pfahl, E., Baganoff, F. K., et al. 2005, ApJL, 622, L113

Narayan, R., \& Yi, I. 1995, ApJ, 452, 710

Negoro, H., Miyoshi, S., Ozawa, H., et al. 2010, in ASP Conf. Ser. 434 Astronomical Data Analysis Software and Systems XIX, ed. Y. Mizumoto, K. Morita, \& M. Ohishi (San Francisco, CA: ASP), 127
Negoro, H., Sasaki, R., Tomida, H., et al. 2016, ATel, 9565, 1

Negoro, H., Serino, M., Mihara, T., et al. 2015, ATel, 7504, 1

Oke, J. B., Cohen, J. G., Carr, M., et al. 1995, PASP, 107, 375

Oudmaijer, R., Groenewegen, M. A. T., \& Schrijver, H. 1999, A\&A, 341, L55

Patruno, A., \& Watts, A. L. 2012, arXiv:1206.2727

Perryman, M. A. C., Lindegren, L., Kovalevsky, J., et al. 1997, A\&A, 323, L49 Pickles, A. J. 1998, PASP, 110, 863

Portegies Zwart, S., van den Heuvel, E. P. J., van Leeuwen, J., \& Nelemans, G. 2011, ApJ, 734, 55

Poznanski, D., Prochaska, J. X., \& Bloom, J. S. 2012, MNRAS, 426, 1465

Pylyser, E., \& Savonije, G. J. 1988, A\&A, 191, 57

Ransom, S. M., Stairs, I. H., Archibald, A. M., et al. 2014, Natur, 505, 520

Rau, A., Yates, R., \& Greiner, J. 2015, ATel, 7524, 1

Roming, P. W. A., Kennedy, T. E., Mason, K. O., et al. 2005, SSR, 120, 95

Schlafly, E. F., \& Finkbeiner, D. P. 2011, ApJ, 737, 103

Shakura, N. I., \& Sunyaev, R. A. 1973, A\&A, 24, 337

Sugimoto, J., Negoro, H., Sugizaki, M., et al. 2015, ATel, 8143, 1

Tanaka, K., Negoro, H., Ueno, S., et al. 2016, ATel, 8529, 1

Tanaka, Y., \& Shibazaki, N. 1996, ARA\&A, 34, 607

van Haaften, L. M., Nelemans, G., Voss, R., et al. 2013, A\&A, 552, A69

van Paradijs, J., \& McClintock, J. E. 1995, in X-ray Binaries, ed

W. H. G. Lewin, J. van Paradijs, \& E. P. J. van den Heuvel (Cambridge: Cambridge Univ. Press), 58

Wood, B. E., Müller, H.-R., Zank, G. P., Linsky, J. L., \& Redfield, S. 2005, ApJL, 628, L143 